\title{
Risk factors associated with health disorders in sport and leisure horses in the Netherlands ${ }^{1}$
}

\author{
E. K. Visser, ${ }^{* 2}$ F. Neijenhuis, ${ }^{*}$ E. de Graaf-Roelfsema, $†$ H. G. M. Wesselink, J. de Boer,§ M. C. van \\ Wijhe-Kiezebrink,* B. Engel,\# and C. G. van Reenen*
}

\begin{abstract}
*Livestock Research, Department of Animal Welfare, Wageningen University and Research Centre, PO Box 65, NL-8200 AB Lelystad, the Netherlands; $\uparrow$ Faculty of Veterinary Medicine, Department of Equine Sciences, Utrecht University, Yalelaan 114, NL-3584 CM Utrecht, the Netherlands; $\$$ Independent Veterinary Professional, Beerzerweg 11, NL-7736 PH Beerze, the Netherlands; §GD Animal Health Service, PO Box 9, NL-7400 AA Deventer, the Netherlands; and \#Biometris, Wageningen University and Research Centre, PO Box 100, NL-6700 AC Wageningen, the Netherlands
\end{abstract}

\begin{abstract}
Horses are used for a wide variety of purposes from being used for recreational purposes to competing at an international level. With these different uses, horses have to adapt to numerous challenges and changes in their environment, which can be a challenge itself in continuously safeguarding their welfare. The objective of this study was to assess the prevalence of health disorders with clinical examination and identify possible risk factors of health disorders affecting horse welfare in professional husbandry systems in the Netherlands. With the use of fixed protocols for recording health aspects in horses, 150 horse farms voluntarily participating in the study were assessed by trained assessors. On each farm, 20 horses were clinically examined, in total almost 3,000 animals. This study recorded on the basis of the clinical examinations: the respiratory system (i.e., abnormal breathing [1\%], coughing [1\%], nasal discharge $[1.9 \%]$ ), body condition (i.e., $18.8 \%$ fat body condition and $6.4 \%$ poor body condition), locomotion (14.5\% exhibited irregularity of locomotion and $4.8 \%$ were lame), back palpation (a light response [22.6\%] and moderate to severe response [8.4\%]), mouth (i.e., irregularities on
\end{abstract}

mouth corners [3.4\%] and bars [3.4\%]), and ocular discharge (12\%). Risk factor analysis, stepwise using mixed model regression, demonstrated several risk factors for health aspects. Horses used for instruction (riding lessons) were almost two times more at risk to develop moderate to severe back pain compared to horses used for recreation (odds ratios $[\mathrm{OR}]=0.54)$ or for competition $(\mathrm{OR}=$ 0.61). Horses used for instruction (riding school lessons), breeding, or recreation all had a higher risk for irregular locomotion or lameness compared to competition horses $(\mathrm{OR}=0.42, \mathrm{OR}=0.55, \mathrm{OR}=2.14$, respectively $)$. Horses used for recreation were more prone to have a higher BCS compared to horses used for breeding $(\mathrm{OR}=3.07)$ and instruction $(\mathrm{OR}=2.06)$. The prevalence of health problems and the identified risk factors are valid for the horses in the present study in which farms voluntarily participated. Furthermore, the results may provide the basis for horse welfare and health programs on farm and horse industry levels. With the development of a valid welfare monitoring system for the horse industry, the welfare of horses can be increased through improving awareness and stimulating changes in management.

Keywords: animal-based parameters, health disorders, horses, risk factors, welfare

J. Anim. Sci. 2014.92:844-855 doi:10.2527/jas2013-6692

\footnotetext{
${ }^{1}$ The present study was funded by the Dutch Ministry of Agriculture and Economic Affairs, the Dutch Society for the Prevention of Cruelty to Animals, the Dutch Federation of Agriculture and Horticulture (LTO), the Royal Dutch Equestrian Federation (KNHS), and the Federation of Dutch Equestrian Entrepreneurs (FNHO). The authors would like to express their gratitude to all farm owners and personnel that participated in this large-scale study and the horse owners who kindly permitted their horses to be used in this study. Moreover, the authors especially thank all students involved in collecting data.

${ }^{2}$ Corresponding author: kathalijne.visser@wur.nl

Received May 12, 2013.

Accepted November 20, 2013.
}

\section{INTRODUCTION}

Keeping horses within a stabled environment, and using them for riding and driving purposes, requires a consideration about how the environment and activities affect their physical and mental welfare. Discomfort or painful conditions can arise through injury associated with inadequate housing, feeding, management, activi- 
ties, and equipment (Casey, 2002). The resulting physical health issues, such as lameness, back pain, and too fat body condition have been reported in countries in which horses are mainly used for competition and for pleasure (Wyse et al., 2008; McGowan et al., 2010; Murray et al., 2010; Ireland et al., 2012; Lesimple et al., 2012). To ensure that there is an acceptable level of horse welfare, an objective assessment or monitoring system is warranted. In developing countries, where working horses are mainly used as a resource for transport, health issues such as body lesions, wounds, poor body condition, and abnormal gait cause decreased welfare. In these countries the health problems are being assessed by direct observations (Pritchard et al., 2005; Burn et al., 2010).

In the European Welfare Quality project an assessment system was developed for multiple species based on direct observations and primarily focusing on the state of the animal rather than just the nature and quality of its living conditions (Blokhuis et al., 2003). This monitoring system focusses on the assessment of animal-based parameters, such as body conditions, wounds, and respiratory problems, in addition to environmental parameters such as housing, bedding, and feeding management. Unfortunately, this monitoring system was not developed for horses yet.

The aim of the present study was to assess the prevalence of health disorders collected with a welfare monitoring system especially developed for horses and identify possible risk factors of health disorders affecting horse welfare in professional husbandry systems in the Netherlands.

\section{MATERIAL AND METHODS}

\section{Farms and Horses}

A cross-sectional study was performed in which the health disorders of horses at riding schools, boarding stables, breeding farms, and sport/training stables were assessed.

The study was performed in the Netherlands during the indoor season, between February and April 2010, and comprised 150 voluntarily participating farms, with a total of 2,956 horses. Farms were recruited by their representing organizations (i.e., equestrian centers, agricultural entrepreneurs, and employers' organizations). Criteria for farms to be selected for this study were 20 horses or more per farm, presence of an employee familiar to the horses and handling all horses during the clinical examinations, and all horses being stabled indoors for at least half of the day.

The average farm size was 61 horses (varying from 8 to 258 horses per farm); farms were situated across the country. The majority of the farms used their horses for riding lessons $(29.9 \%$ of the farms), followed by recreation $(26.2 \%$ of the farms), and competition $(22.8 \%$ of the farms). The gender of the horses was $50 \%$ mares, $44.2 \%$ geldings and $5.8 \%$ stallions. The average age of the horses was $11 \mathrm{yr}$ (varying from $1 \mathrm{yr}$ to $40 \mathrm{yr}$ of age). Most horses were housed in single stalls (83.4\%), 13.8\% in groups ( 2 or more horses), $2.3 \%$ in standing or tie stalls, and $0.4 \%$ in other types of housing systems.

\section{Parameters}

Before this study, a welfare assessment protocol was developed for horses following the Welfare Quality protocols for farm animal species. The protocol for horses included animal-based measures as well as resource- and management-based parameters related to the 4 principles used in the Welfare Quality framework: feeding, housing, health, and behavior. The protocol can be downloaded at http://edepot.wur.nl/238619.

The main focus of the welfare assessment involved the clinical examinations in which health parameters were evaluated (Table 1).

Respiratory problems are closely associated with housing and the presence of dust and fungal spores in the air (Halliwell et al., 1993) and can result in a range of signs including coughing, abnormal breathing, nasal discharge, and ocular discharge. Stable hygiene practices (i.e., raised ammonia levels) and bedding choice (i.e., straw) can cause respiratory problems (Clarke, 1987).

It is commonly known that a low BCS can have detrimental effects on animal welfare (Pritchard et al., 2005; Burn et al., 2010). However, there is also growing evidence that obesity due to over nutrition among horses is increasing (Lesimple et al., 2010; Ireland et al., 2012) and represents a significant detriment to health of equines in a manner similar to that by which it is affecting the human population. Studies have revealed varying prevalences up to $45 \%$ in pleasure riding horses in the UK (Wyse et al., 2008). Obesity promotes insulin resistance, and insulin resistance plays a role in the pathogenesis of laminitis, a potentially severe and debilitating cause of lameness in the equine species (Frank, 2009; Johnson et al., 2009). NEWC (2009) stated in 2009 that a BCS of 5 in the horse is a welfare issue and should be avoided in any horse. Not only is a BCS of 5 causing health problems, a BCS of 4 is also associated with increased risk of developing laminitis, as reported by Carter et al. (2009).

Pathological or painful conditions such as lameness, sore mouth, and back pain can arise through injury associated with inadequate housing, management, activities, and equipment (Wyse et al., 2008; Lesimple et al., 2010; McGowan et al., 2010; Murray et al., 2010; Ireland et al., 2012; Lesimple et al., 2012). Lameness was a frequently reported health problem by owners of dressage horses in the study of Murray et al. (2010): 33\% of the horses were lame at some time during their career. Other studies report $13 \%$ 
Table 1. Animal-based parameters, the assessment method and the classification ${ }^{1}$

\begin{tabular}{|c|c|c|}
\hline Areas of concern & Assessment method & Classification \\
\hline \multicolumn{3}{|l|}{ Respiratory problems } \\
\hline Abnormal breathing & Evaluation of the breathing manner: increase in depth and effort of breathing & No $\mid$ Yes \\
\hline Nasal discharge & $\begin{array}{l}\text { Evaluation of the nasal discharge: seromucous, } \\
\text { purulent, food or hemorrhagic/blood were considered as abnormal }\end{array}$ & No $\mid$ Yes \\
\hline $\mathrm{BCS}$ & $\begin{array}{l}\text { BCS is evaluated with the Carroll and Huntington (1988) } \\
\text { scale 1-5, with 1-2 indicating a poor body condition, } 3 \text { normal, 4-5 fat/obese }\end{array}$ & BCS $1-5$ \\
\hline Back pain & $\begin{array}{l}\text { Evaluation of the response to manually superficial and deep } \\
\text { palpation of back musculature and spinal processus of thoracolumbal spine }\end{array}$ & $\begin{array}{c}\text { No disorders | sensitive/tense | painful } \\
\text { meaning moderate to serious (behavioural) } \\
\text { responses on pressure like kicking, biting etc. }\end{array}$ \\
\hline \multicolumn{3}{|l|}{ Mouth } \\
\hline
\end{tabular}

${ }^{1}$ Full protocol can be downloaded at http://edepot.wur.n1/238619.

(Cole et al., 2005), 23\% (Ireland et al., 2012), and 28\% lameness (McGowan et al., 2010). In the same study of Murray et al. (2010), 25\% of dressage horses were reported to have a back pain problem by their owners. Other studies estimate the prevalence of back disorders to lie between 3.9 and $22 \%$ using questionnaire surveys (Lesimple et al., 2012).

\section{Sampling}

On each farm, horses of a randomly selected group of 20 animals were assessed individually. Selection of horses was done before the actual assessment with the help of a plan of the farm indicating housing systems and corresponding numbers of horses present. On farms with less than 20 horses in total ( $n=16$ farms), all animals were observed. Within the same farm, the sample of horses was evenly distributed among individual and group housing systems if both were present. During the farm visits, each assessor was accompanied by an assistant to record the results into specially designed software on a personal digital assistant device. The total assessment per farm lasted $6 \mathrm{~h}$ on average, of which the clinical examination took approximately $10 \mathrm{~min}$ per horse. Besides the clinical examinations, the behavior, stall climate, and the stable management were assessed. The farm visits were executed on normal working days and started around $0900 \mathrm{~h}$, after the first feeding time. The assessor and assistant entered the farm in clean outfit and used disinfection spray for the hands to minimize risk of spreading contagious diseases.

\section{Clinical Examinations}

Assessment started with examining breathing of the horses in their home stable. Thereafter, horses were hal- tered and examined farther outside the stable in a place with enough space and light.

The assessment was performed by 5 fifth-year veterinary students. The veterinary students were trained to perform the clinical examinations by two senior veterinarians. Both senior veterinarians had over $5 \mathrm{yr}$ of experience. One of them had experience as an independent veterinary professional at international (FEI) competitions; the other veterinarian was appointed as clinical professional at the Veterinary Faculty of Utrecht University. Before training the students, the inter- and intrareliability of the senior veterinarians was checked (interobserver reliability kappa $\geq 0.39$, and intraobserver reliability kappa $\geq 0.37$ ). The assessors were trained 2 mo before the assessments at similar farms, constituting a total of 90 horses with different health disorders. All students assessed the same 90 horses. Training consisted of $1 \mathrm{~d}$ of judging a total of 120 photos of healthy horses and horses with health disorders (phase 1). This did not include training of parameters that warranted "live horses," such as training for the assessment of lameness or abnormal breathing. These photos were scored by the senior veterinarians as golden standards before the training. The second phase of training consisted of 7 full days of farm visits in which clinical parameters of the protocol were trained. The training was considered completed when the students reached the criteria of $\geq 80 \%$ agreement with the outcome of the golden standard, i.e., photos (phase 1), and moderate to excellent agreement with the silver standards, i.e., senior veterinarians (kappa value $\geq 0.41$; Burn et al., 2009) for each parameter to be assessed in the second phase. After the successful completion of the training, students were promoted to "assessors." 
The veterinary students were also trained to take the necessary precaution when handling the horses to minimize the risk of harm to the horses and the people involved. An employee of the stable was present, and the horse was always approached from the front. When a horse showed extreme fear or aggression or was not accustomed to handling or haltering, the assessment that may imply any risk of harm to the animal, handler, or assessor was skipped.

\section{Farm Environment, Housing, and Management}

Specific data regarding the farm (i.e., type of farm), housing (i.e., individual, groups, area per horse, social contact), feeding (i.e., roughage, concentrates), climate (i.e., temperatures, relative air humidity, wind speed), and management (i.e., use of horses, health care), were gathered from an interview with the stockperson and/or by observation and assessment by the assessor. Detailed information can be found in the protocol.

\section{Data Processing and Statistical Analysis}

Raw data gathered during the assessment and from the interviews were saved in an Access database. The database was checked for irregularities and missing data.

Descriptive analyses were performed on both animal-based and resource- and management-based parameters. All potential risk factors were defined as discrete (class) parameters. For continuous resource- and management-based parameter thresholds and classes were chosen according to biological relevance and in such a way that sufficient numbers of animals were present in each class. An overview of potential risk factors and corresponding classes is given in Table 2.

Basic records in the database were individual horses. In the final analysis each clinical measure was expressed at the level of the individual horse as a binary variable with 2 possible outcomes: 1 (i.e., the clinical disorder was present) or 0 (i.e., the clinical problem was absent). Thus, for multiclass variables (i.e., BCS, locomotion, and back pain; see Table 1), multiple categories were summarized into single ones as follows. Body condition score was expressed as either not fat (too poor, poor, or normal) or fat (fat or too fat), locomotion was expressed as either normal (no irregularities) or abnormal (irregular or lame), and back pain was expressed as either no disorders present (no disorders) or disorders present (sensitive/tense or painful).

Risk factor analysis was performed only for health disorders that showed an overall prevalence $\geq 3 \%$. Prevalences of disorders were considered as response variables $(\mathrm{Y})$, and the management-based variables $(\mathrm{X})$ were the potential risk factors. To examine the relation- ship between clinical problems and potential risk factors, stepwise multivariate logistic regression analysis was used. To account for dependence between horses from the same farm, random farm effects were introduced in the regression model, resulting in a generalized linear mixed model (GLMM). Random effects for farms and fixed effects for potential risk factors were introduced on the logit scale, and conditional on the random farm effects a binomial variance function was assumed. Data from the GLMM were analyzed by iterative re-weighted restricted maximum likelihood (Searle et al., 1992; Breslow and Clayton, 1993; Engel and Keen, 1994).

Risk factor analysis involved a series of successive steps. First, each potential risk factor was introduced as a single explanatory factor in a GLMM. Risk factors with a $P$-value below 0.15 from the F-test (Kenward and Roger, 1997) were considered for further multivariate analysis. Next, forward and backward selection was performed, with preset bounds for the F-tests for inclusion and exclusion of potential risk factors. Only main effects were considered in this step. For the risk factors selected in either of the two selection approaches an all possible subset selection was performed, where models with one risk factor, with two risk factors, etc. were ranked with respect to their mean deviance (Goedhart, 2011). Only risk factors that contributed significantly $(P<0.05$ after an F-test) were retained in the final step. The statistical procedure included a specific routine for the assessment of (partial) confounding of factors, i.e., of potential risk factors that are (partially) correlated. The aim was to present a final model without (partially) confounded factors. The final model was fitted to the data. Predicted means and associated standard errors were evaluated. For each risk factor retained in the final model, odds ratios (OR) as well as $95 \%$ confidence intervals were obtained.

All the calculations were performed within GenStat (VSNInternational, 2012).

\section{RESULTS}

\section{Prevalence of Respiratory Disorders}

For respiratory disorders, the proportion of horses with abnormal breathing was $1 \%$ (29 out of 2,894 horses), with ample nasal discharge was $1.9 \%$ (57 out of 2,955 horses), and the proportion of horses coughing during the 10-min monitoring period was $1 \%$ (30 out of 2,956 horses).

\section{Prevalence of and Risk Factors for Horses with Abnormal Body Condition}

Body condition score was given to 2,939 horses using the Carroll and Huntington (1988) scoring method. 
Table 2. Management- and resource-based parameters included in the final model of the risk factor analysis

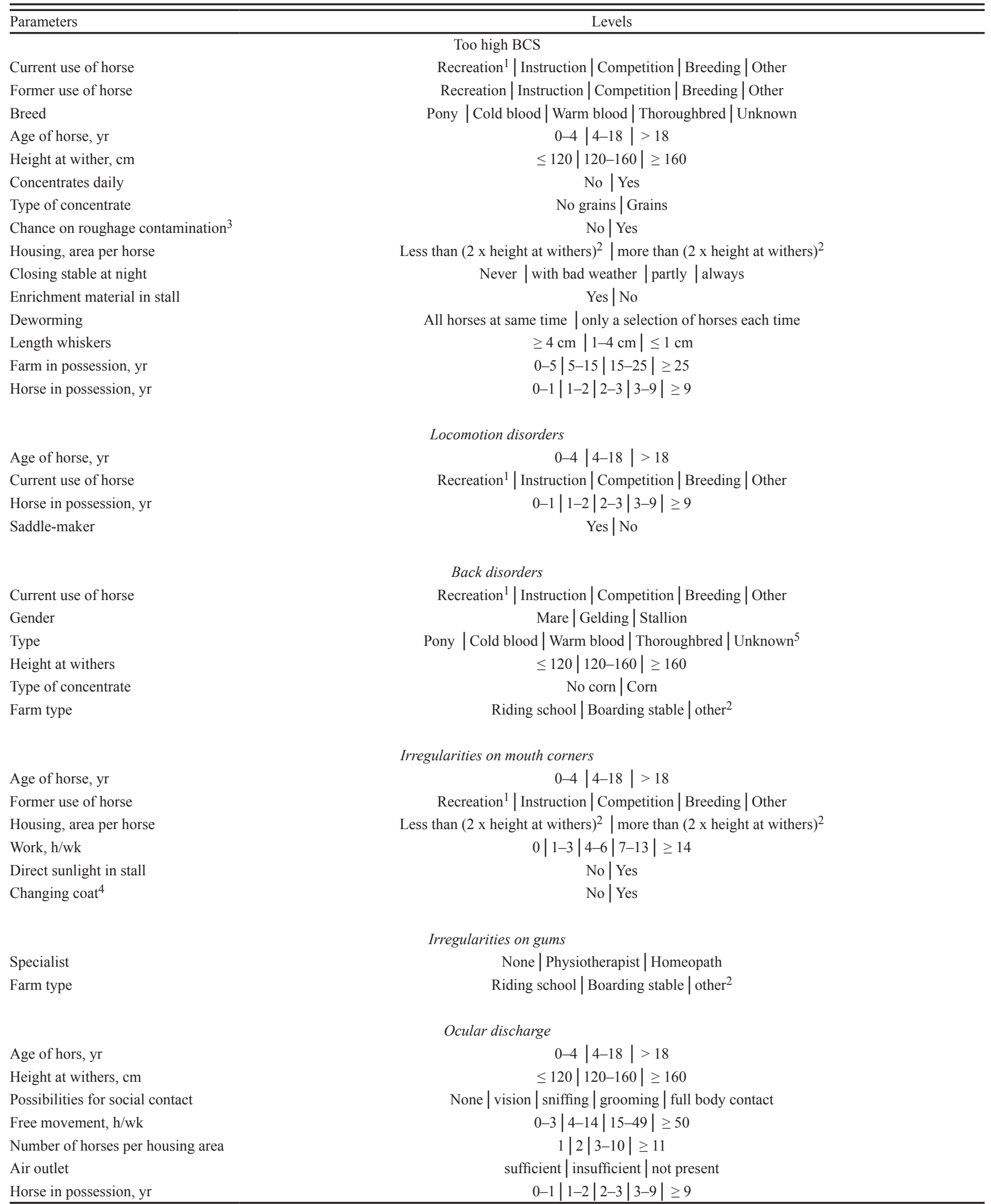

${ }^{1}$ Includes horses kept as companion animal.

${ }^{2}$ Includes farms for breeding, training, competition.

${ }^{3}$ Roughage may get contaminated when it is provided on the ground within the area the horse is stabled.

${ }^{4}$ Severe (normal) shedding of the coat through which the body condition cannot be assessed easily.

${ }^{5}$ Type of the horse as determined according to their breed. 
Table 3. Body condition score horses ${ }^{1}$

\begin{tabular}{lcc}
\hline \hline BCS & Number of horses & Percentage \\
\hline 1. Very poor & 5 & 0.2 \\
2. Poor & 188 & 6.4 \\
3. Normal & 2195 & 74.7 \\
4. Fat & 528 & 18.0 \\
5. Very Fat & 23 & 0.8 \\
Total & 2939 & 100.1 \\
\hline
\end{tabular}

${ }^{1}$ Method of Carroll and Huntington (1988).

$74.7 \%$ of horses received a normal score for body condition (score 3), $18.8 \%$ of the horses received a score above 3 , and $6.4 \%$ of the horses were assessed as poor (score 2 ) or very poor (score 1 ; Table 3 ).

Table 4 shows the risk factors for obesity or increased body condition (BCS of 4 or 5). Sixteen variables were significantly associated with the occurrence of obesity or increased body condition. Horses used for recreation were more prone to have a higher BCS compared to horses used for breeding and instruction; horses used in competition were more at risk to develop a higher BCS compared to horses used for breeding. Middleaged horses (4-18 yr) were more prone to have a higher body condition compared to horses over $18 \mathrm{yr}$ old. Smaller horses were more at risk to have a high body condition compared to taller horses. Correspondingly, ponies were more prone to develop a higher body condition compared to warm bloods and thoroughbreds; cold bloods were more prone to develop a higher body condition compared to thoroughbreds. Horses housed in individual stalls with an area of less than $(2 \times$ height at the withers $)^{2}$ were less prone to the risk of a high body condition compared to horses with a larger stall area. Horses fed concentrates on a daily basis were less at risk to have a higher body condition compared to horses that were not fed concentrates on a daily basis.

\section{Prevalence of and Risk Factors for Lameness}

From the 2,566 horses that were checked for lameness, $14.5 \%$ appeared to be irregular (i.e., stiff, short lo-

Table 4. Final multivariate logistic regression model for the occurrence of a (too) fat body condition (BCS 4 or BCS 5)

\begin{tabular}{|c|c|c|c|c|c|}
\hline Risk factor $^{\mathrm{a}}$ & $P$-value & Level 1 & Level 2 & Odds ratio $^{\mathrm{b}}$ & Confidence interval \\
\hline \multirow[t]{3}{*}{ Current use of horse } & \multirow[t]{3}{*}{$<0.001$} & Recreation & Breeding & 3.07 & $1.59-5.92$ \\
\hline & & Recreation & Instruction & 2.06 & $1.46-2.91$ \\
\hline & & Competition & Breeding & 2.05 & $1.02-4.10$ \\
\hline Former use horse breeding & 0.042 & No & Yes & 1.49 & $1.01-2.20$ \\
\hline Former use horse competition & 0.017 & No & Yes & 1.52 & $1.06-2.18$ \\
\hline \multirow[t]{3}{*}{ Type } & \multirow[t]{3}{*}{0.003} & Pony & Warm blood & 1.58 & $1.08-2.31$ \\
\hline & & Pony & Thoroughbred & 3.07 & $1.39-6.76$ \\
\hline & & Cold blood & Thoroughbred & 4.12 & $1.31-12.99$ \\
\hline Age of horse, yr & 0.008 & $4-18$ & $>18$ & 1.77 & $1.21-2.60$ \\
\hline \multirow[t]{3}{*}{ Height at withers, $\mathrm{cm}$} & \multirow[t]{3}{*}{$<0.001$} & $<120$ & $120-160$ & 1.91 & $1.07-3.40$ \\
\hline & & $<120$ & $>160$ & 3.73 & $1.81-7.67$ \\
\hline & & $120-160$ & $>160$ & 1.96 & $1.34-2.86$ \\
\hline Concentrates on a daily basis & 0.003 & No & Yes & 1.75 & $1.22-2.51$ \\
\hline Type of concentrate & 0.010 & No grains & Grains & 2.45 & $1.23-4.89$ \\
\hline Chance on roughage contamination & $<0.001$ & No & Yes & 0.45 & $0.30-0.70$ \\
\hline Housing, area per horse & 0.025 & Less than (2xheight at the withers $)^{2}$ & More than (2xheight at the withers $)^{2}$ & 0.70 & $0.52-0.96$ \\
\hline \multirow[t]{2}{*}{ Closing stable at night } & \multirow[t]{2}{*}{0.038} & With bad weather & Partly & 0.60 & $0.38-0.93$ \\
\hline & & With bad weather & Never & 0.56 & $0.33-0.95$ \\
\hline Enrichment in stall & 0.003 & Yes & No & 1.49 & $1.14-1.94$ \\
\hline Deworming & 0.020 & All horses at same time & Selection & 0.46 & $0.24-0.90$ \\
\hline \multirow[t]{2}{*}{ Length whiskers, $\mathrm{cm}$} & \multirow[t]{2}{*}{0.020} & $>4$ & $1-4$ & 1.61 & $1.06-2.44$ \\
\hline & & $>4$ & $<1$ & 2.07 & $1.01-4.25$ \\
\hline \multirow[t]{2}{*}{ Farm in possession, $\mathrm{yr}$} & \multirow[t]{2}{*}{0.013} & $\geq 25$ & $0-5$ & 0.42 & $0.23-0.75$ \\
\hline & & $15-25$ & $0-5$ & 0.52 & $0.31-0.86$ \\
\hline \multirow[t]{5}{*}{ Horse in possession, yr } & \multirow[t]{5}{*}{$<0.001$} & 0 & $>9$ & 0.42 & $0.20-0.89$ \\
\hline & & 1 & $>9$ & 0.40 & $0.25-0.64$ \\
\hline & & 2 & $3-9$ & 0.61 & $0.39-0.93$ \\
\hline & & 2 & $>9$ & 0.31 & $0.19-0.52$ \\
\hline & & $3-9$ & $>9$ & 0.51 & $0.35-0.74$ \\
\hline
\end{tabular}

${ }^{\mathrm{a} A}$ All risk factors in the final model were significant $(P<0.05)$.

${ }^{b}$ Odds Ratios (ORs) are given for pairwise comparisons between classes (levels) of risk factors. Level 2 is the reference level. When value 1 is not part of the 95\% confidence interval, the corresponding OR is significant $(P<0.05)$. When $\mathrm{OR}<1$ the risk is decreased in comparison with the reference level. When OR $>$ 1 the risk is increased in comparison with the reference level. 
Table 5. Final multivariate logistic regression model for irregular locomotion or lameness

\begin{tabular}{|c|c|c|c|c|c|}
\hline Risk factor $^{\mathrm{a}}$ & $P$-value & Level 1 & Level 2 & Odds ratio $^{b}$ & Confidence interval \\
\hline Age of horse, $\mathrm{yr}$ & $<0.001$ & $0-4$ & $4-18$ & 0.16 & $0.06-0.38$ \\
\hline \multirow[t]{2}{*}{ Current use of horse } & $<0.001$ & Recreation & Competition & 2.14 & $1.49-3.07$ \\
\hline & & Competition & Instruction & 0.42 & $0.29-0.61$ \\
\hline \multirow[t]{3}{*}{ Horse in possession, $\mathrm{yr}$} & $<0.001$ & $1-2$ & $2-3$ & 1.59 & $1.01-2.51$ \\
\hline & & $1-2$ & $>9$ & 0.59 & $0.40-0.87$ \\
\hline & & $3-9$ & $>9$ & 0.63 & $0.47-0.84$ \\
\hline Saddle-maker & 0.003 & No & Yes & 1.62 & $1.20-2.90$ \\
\hline
\end{tabular}

${ }^{\mathrm{a}}$ All risk factors in the final model were significant $(P<0.05)$.

${ }^{\mathrm{b}}$ Odds Ratios (ORs) are given for pairwise comparisons between classes (levels) of risk factors. Level 2 is the reference level. When value 1 is not part of the $95 \%$ confidence interval, the corresponding OR is significant $(P<0.05)$. When $\mathrm{OR}<1$ the risk is decreased in comparison with the reference level. When OR $>$ 1 the risk is increased in comparison with the reference level.

comotion; not possible to point out which leg is causing the irregular motion). Another $4.8 \%$ of all horses appeared to be lame. Risk factor analyses showed that age, current use of horse, number of years the horse was in possession of current owner, and whether or not the farm consults a saddle maker were significantly associated with lameness (Table 5). In comparison with older horses, younger horses had a lower risk to be lame. Horses used for instruction (riding school lessons), breeding, or recreation all had a higher risk for irregular locomotion or lameness compared to competition horses. In this data set, horses housed at a farm that consults a saddle maker for their horses at regular intervals were more at risk to develop locomotion disorders compared to farms who did not consult a saddle maker.

\section{Prevalence of and Risk Factors for Back Pain}

After manual back palpation, horses were classified as no response on back palpation, slight response (behavioral reaction and tense back muscles), and moderate to severe response (behavioral reaction, i.e., aggressive or retreat). From the 2,824 horses that were checked for a response on back palpation, the percentage of horses with no response on back palpation was $68.9 \%$, with a slight response was $22.6 \%$, and with moderate to severe response was $8.4 \%$. Risk factor analyses revealed that cur-

Table 6. Final multivariate logistic regression model for back pain disorders

\begin{tabular}{|c|c|c|c|c|c|}
\hline Risk factor $^{\mathrm{a}}$ & $P$-value & Level 1 & Level 2 & Odds ratio $^{b}$ & Confidence interval \\
\hline \multirow[t]{8}{*}{ Current use of horse } & $<0.001$ & Recreation & Breeding & 3.41 & $2.02-5.75$ \\
\hline & & Recreation & Instruction & 0.54 & $0.41-0.70$ \\
\hline & & Recreation & Other & 2.70 & $1.30-5.62$ \\
\hline & & Competition & Breeding & 3.87 & $2.28-6.56$ \\
\hline & & Competition & Instruction & 0.61 & $0.45-0.82$ \\
\hline & & Competition & Other & 3.07 & $1.49-6.33$ \\
\hline & & Breeding & Instruction & 0.16 & $0.09-0.27$ \\
\hline & & Instruction & Other & 5.05 & $2.43-10.52$ \\
\hline Gender & 0.013 & Mare & Gelding & 1.28 & $1.04-1.57$ \\
\hline \multirow[t]{3}{*}{ Breed } & 0.021 & Pony & Thoroughbred & 0.46 & $0.26-0.79$ \\
\hline & & Cold blood horse & Thoroughbred & 0.27 & $0.09-0.80$ \\
\hline & & Warm blood horse & Thoroughbred & 0.49 & $0.28-0.84$ \\
\hline \multirow[t]{3}{*}{ Height at withers, $\mathrm{cm}$} & $<0.001$ & $<120$ & $120-160$ & 0.28 & $0.14-0.56$ \\
\hline & & $<120$ & $>160$ & 0.14 & $0.07-0.29$ \\
\hline & & $120-160$ & $>160$ & 0.50 & $0.38-0.67$ \\
\hline Type of concentrate & 0.004 & No corn & Corn & 0.54 & $0.35-0.83$ \\
\hline \multirow[t]{2}{*}{ Farm type } & 0.004 & Riding school & Other & 2.87 & $1.55-5.30$ \\
\hline & & Boarding stable & Other & 2.54 & $1.43-4.81$ \\
\hline
\end{tabular}

${ }^{\mathrm{a} A l l}$ risk factors in the final model were significant $(P<0.05)$.

${ }^{\mathrm{b}}$ Odds Ratios (ORs) are given for pairwise comparisons between classes (levels) of risk factors. Level 2 is the reference level. When value 1 is not part of the 95\% confidence interval, the corresponding OR is significant $(P<0.05)$. When OR $<1$ the risk is decreased in comparison with the reference level. When OR $>$ 1 the risk is increased in comparison with the reference level. 
Table 7. Final multivariate logistic regression model for irregularities at mouth corners

\begin{tabular}{|c|c|c|c|c|c|}
\hline Risk factor $^{\mathrm{a}}$ & $P$-value & Level 1 & Level 2 & Odds ratio $^{b}$ & Confidence interval \\
\hline \multirow[t]{3}{*}{$\overline{\text { Age, } y r}$} & $<0.001$ & $0-4$ & $4-18$ & 0.09 & $0.01-0.70$ \\
\hline & & $0-4$ & $>18$ & 0.06 & $0.01-0.45$ \\
\hline & & $4-18$ & $>18$ & 0.64 & $0.49-0.84$ \\
\hline Housing, area per horse & 0.007 & $\begin{array}{l}\text { Less than (2xheight at } \\
\text { withers })^{2}\end{array}$ & $\begin{array}{c}\text { More than }(2 \mathrm{xheight} \text { at } \\
\text { withers })^{2}\end{array}$ & 1.42 & $1.13-1.77$ \\
\hline \multirow[t]{8}{*}{ Work, h/wk } & $<0.001$ & 0 & $1-3$ & 0.40 & $0.20-0.81$ \\
\hline & & 0 & $4-6$ & 0.35 & $0.19-0.62$ \\
\hline & & 0 & $7-13$ & 0.22 & $0.13-0.40$ \\
\hline & & 0 & $\geq 14$ & 0.16 & $0.08-0.31$ \\
\hline & & $1-3$ & $7-13$ & 0.56 & $0.33-0.94$ \\
\hline & & $1-3$ & $\geq 14$ & 0.40 & $0.22-0.73$ \\
\hline & & $4-6$ & $7-13$ & 0.65 & $0.50-0.84$ \\
\hline & & $4-6$ & $\geq 14$ & 0.46 & $0.30-0.71$ \\
\hline
\end{tabular}

aAll risk factors in the final model were significant $(P<0.05)$.

bOdds Ratios (ORs) are given for pairwise comparisons between classes (levels) of risk factors. Level 2 is the reference level. When value 1 is not part of the $95 \%$ confidence interval, the corresponding OR is significant $(P<0.05)$. When $\mathrm{OR}<1$ the risk is decreased in comparison with the reference level. When $\mathrm{OR}>1$ the risk is increased in comparison with the reference level.

rent use of the horse, gender, type, height at the withers, type of concentrate, and farm type were all significantly associated with back pain (slight or moderate to severe response on back palpation); see Table 6 . Horses used for instruction (riding lessons) were almost 2 times more at risk compared to horses used for recreation $(\mathrm{OR}=0.54)$ or for competition $(\mathrm{OR}=0.61)$. Horses used for competition, instruction, or recreation were more at risk compared to horses used for breeding. In comparison with geldings, mares had a higher risk for back pain. Of the various type of horses included in this data set, thoroughbreds were the most at risk for back pain. In addition, taller horses were more prone to the risk of back pain than smaller ones.

\section{Prevalence of and Risk Factors for Irregularities in the Mouth Corners}

Irregularities that were noted in the mouth corners were hardened spots, redness, and (open) wounds. From the 2,717 horses that were checked for irregularities at the mouth corners, the percentage of horses without irregularities was $81.5 \%$ and with irregularities was $18.5 \%$. Risk factor analysis revealed that 3 variables were significantly associated with irregularities in the mouth corners, including age, area per horse, and quantity of work (Table 7). Younger horses were less at risk compared to older ones, and horses housed in an area less than $(2 \times \text { height at the withers })^{2}$ were more at risk compared to horses housed in an area more than $(2 \times$ height at the withers $)^{2}$. It seems unlikely that the area per horse directly influenced the condition of the mouth corners. Perhaps the area per horse was associated with different types of work the horse was used for. Finally, the more hours horses had to work during the week, the higher the risk for irregularities in the mouth corners.

\section{Prevalence of and Risk Factors \\ for Irregularities at the Gums}

As for the mouth corners, irregularities at the gums were noted when hardened spots, redness, and or (open) wounds were found. A small percentage $(3.4 \%)$ of a total of 2,717 horses showed irregularities; $96.6 \%$ of the horses had no irregularities at the gums. Risk factor analyses revealed that horses housed in a boarding stable were almost 4 times more at risk $(\mathrm{OR}=3.77$, confidence interval 1.14-12.49) compared to horses housed in breeding stations or competition/training stables $(P=0.044)$.

\section{Prevalence of and Risk Factors for Ocular Discharge}

Twelve percent of the total of 2,854 horses assessed for ocular discharge were found with ocular discharge. Table 8 summarizes the significant risk factors for ocular discharge. In general, older horses $(>18 \mathrm{yr})$ were more at risk compared to younger horses $(0-4 \mathrm{yr}$ of age and 4-18 yr of age). With more horses in indoor housing, the risk for the development of ocular discharge increased, although housing in groups with $\geq 11$ animals reduced the risk for ocular discharge in comparison with group sizes from 2-10 horses. Similarly, the more hours horses spent outside the stall for free movement (paddock of pasture) the higher the risk for ocular discharge. It was shown that the absence of an air outlet was associated with a reduced risk for ocular discharge in comparison with a situation of sufficient air outlet. 
Table 8. Final multivariate logistic regression model for ocular discharge

\begin{tabular}{|c|c|c|c|c|c|}
\hline Risk factor $^{\mathrm{a}}$ & $P$-value & Level 1 & Level 2 & Odds ratio $^{b}$ & Confidence interval \\
\hline \multirow[t]{2}{*}{$\overline{\text { Age, } y r}$} & $<0.001$ & $0-4$ & $>18$ & 0.30 & $0.17-0.55$ \\
\hline & & $4-18$ & $>18$ & 0.35 & $0.26-0.48$ \\
\hline \multirow[t]{2}{*}{ Height at the withers, $\mathrm{cm}$} & 0.016 & $<120$ & $120-160$ & 1.87 & $1.08-3.24$ \\
\hline & & $<120$ & $>160$ & 2.29 & $1.28-4.10$ \\
\hline \multirow{2}{*}{$\begin{array}{l}\text { Possibilities for social } \\
\text { contact }\end{array}$} & 0.012 & Vision & Sniffing & 1.45 & $1.05-1.99$ \\
\hline & & Sniffing & Grooming & 0.51 & $0.32-0.82$ \\
\hline \multirow{2}{*}{ Free movement, $\mathrm{h} / \mathrm{wk}$} & & $0-3$ & $\geq 50$ & 0.38 & $0.22-0.64$ \\
\hline & & $4-14$ & $\geq 50$ & 0.53 & $0.33-0.84$ \\
\hline \multirow{3}{*}{$\begin{array}{l}\text { Number of horses per } \\
\text { housing area }\end{array}$} & 0.001 & 1 & 2 & 0.45 & $0.22-0.92$ \\
\hline & & 1 & $3-10$ & 0.39 & $0.18-0.87$ \\
\hline & & 2 & $\geq 11$ & 4.39 & $1.46-13.17$ \\
\hline \multirow{3}{*}{ Horse in possession, $\mathrm{yr}$} & & $1-2$ & $2-3$ & 1.97 & $1.17-3.31$ \\
\hline & & $2-3$ & $3-9$ & 0.50 & $0.30-0.81$ \\
\hline & & $2-3$ & $>9$ & 0.39 & $0.23-0.67$ \\
\hline
\end{tabular}

a All risk factors in the final model were significant $(P<0.05)$.

${ }^{\mathrm{b}}$ Odds Ratios (ORs) are given for pairwise comparisons between classes (levels) of risk factors. Level 2 is the reference level. When value 1 is not part of the 95\% confidence interval, the corresponding OR is significant $(P<0.05)$. When OR $<1$ the risk is decreased in comparison with the reference level. When $\mathrm{OR}>1$ the risk is increased in comparison with the reference level.

\section{DISCUSSION}

Large sample studies in horse welfare and health are very scarce, since budgets and available facilities are limited. Instead, to reach large sample sizes most studies rely on questionnaires sent to owners or caretakers or self-reports (Hotchkiss et al., 2007; Murray et al., 2010; Ireland et al., 2012; Lesimple et al., 2012). However, several authors have shown that the effectiveness of this method is debatable since the response rate may be positively biased (e.g., toward less problems) and owner or caretakers may severely underestimate possible health and welfare problems. To the knowledge of the authors, this has been the first large-scale study in which clinical health disorders affecting horse welfare are being assessed with a standardized method (protocol).

In the present study, estimates of prevalences of clinical problems were based on actual clinical observations in a large sample of randomly selected horses across a range of voluntarily participating farms. The farms volunteering in this study may reflect the characteristics of the entire national farm population with regard to average horses or stabling conditions, although it may be possible that results on clinical problems were positively biased.

In addition to animal-based measures, various management- and resource-based variables (related to housing, feeding, and management) were also systematically recorded. This allowed us to identify potential risk factors using statistical methods that are generally used in veterinary epidemiology (e.g., Cole et al., 2005; Robinson et al. 2006). However, it should be noted that epidemiological research allows us to identify associations between clinical measures and potential risk factors, and that such associations do not necessarily refer to causal relationships.

Respiratory problems are closely associated with housing and the presence of dust and fungal spores in the air (Halliwell et al., 1993). Also, seasonality plays a role in certain respiratory disorders with increased risk factors for detecting inflammatory airway disease in winter and spring compared to summer (Couëtil et al., 2007); inflammatory airway disease was more prevalent in horses kept outside in winter compared to stabled horses (Robinson et al., 2006). In the present study, horses were examined between January and April and were all stabled for a large part of the day. Prevalences of respiratory problems were low $(<3.0 \%)$. The results in this study are far lower compared with results in other studies. In a study by Wheeler et al. (2002), it was reported that $16.9 \%$ of pony club horses in the UK coughed severely during exercise and coughed at least once a week. Furthermore, Hotchkiss et al. (2007) reported that $14 \%$ of general horse population in the UK demonstrated recurrent airway obstruction (RAO). The observation period of $10 \mathrm{~min}$ in the present study has probably been too short to identify the real prevalence of coughing horses in a stable since most horses with chronic respiratory disorders cough, especially during 
exercise. Only severely affected horses with chronic obstructive pulmonary disease do show signs of dyspneu and abnormal breathing. Therefore, most cases of inflammatory airway disease and RAO are subclinical at rest and show more signs during exercise (Couëtil et al., 2007). So, although prevalence of respiratory disorders seem to be low in this study, only severely affected cases were found. Because the prevalence of respiratory disorders found in the present study appeared to be too low (defined as $<3 \%$ ) these have not been included in the risk factor analysis.

The body condition of horses constitutes, as in other companion animal species (German, 2010; Bland and Hill, 2011), a welfare concern. NEWC (2009) stated in 2009 that a horse's body condition should ideally be maintained at BCS 3, varying no more than 2.5 to 3.5 on a scale of 0 (very poor) to 5 (very fat). If a horse's BCS declines to 2 or below or increases to 4 or above, then action should be taken to correct this. A body condition score of 5 is considered to be a welfare problem and should be avoided in every horse (NEWC, 2009). Excessive body fat, which can be local or more generally distributed, can cause impairment to health or bodily function and is associated with insulin resistance, colic, and loss of performance, among others (Geor, 2008). The most widely recognized consequence in horses is laminitis, a painful condition of the feet (Carter et al., 2009; Becvarova and Pleasant, 2012; Galantino-Homer and Engiles, 2012). Both BCS 4 and 5 have been shown to be a reliable predictor of pasture-associated laminitis in a cohort of ponies (Carter et al., 2009).

A prevalence of $18.8 \%$ of horses with an increased body condition found in the present study falls in the wide range (3-45\%) of questionnaire reported results in other studies (i.e., Wyse et al., 2008), but is higher than the $10 \%$ of overweight horses $(\mathrm{BCS}>3)$ that were clinically examined in the study of Ireland et al. (2012). The horses in the study of Ireland et al. (2012) were mainly retired horses ( $>30 \mathrm{yr}$ of age) and hence a lower percentage of overweight horses could have been expected because geriatric horses in general require more nutrition or a diet adapted to their wearing of teeth and increased incidences of diseases such as pituitary pars intermedia dysfunction (PPID; 34\% of the horses older than $30 \mathrm{yr}$ suffered from PPID in the study of Ireland et al. [2012]) and chronic lameness (97\% of the horses older than $30 \mathrm{yr}$ had at least decreased range of motion in one joint [Ireland et al., 2012]), which makes it even more difficult to maintain an acceptable BCS. As was shown in other studies, smaller horses, ponies, and socalled "easy keepers" are more at risk (Frank, 2009), as are recreational horses (Thatcher et al., 2012).

Interestingly, horses housed in smaller stalls were less at risk, and, similarly, horses fed concentrates on a daily basis were less at risk for developing an increased body condition (fat or obese). It could be inferred that this group of horses was exercised more frequently, and because of this, another factor or complex of factors were involved. It needs to be stressed that in this epidemiological study no causal relations can be drawn between environment (size of stalls) and management (feeding concentrates) and the BCS.

In the present study, $19.3 \%$ of the horses appeared to have some sort of irregular gait or were obviously lame. In survey studies, a lameness percentage of $23-33 \%$ was reported by owners, self-reporting the lameness of their horses over a time period of 12 yr (Murray et al., 2010; Ireland et al., 2012). Furthermore, at a specific moment in time, $13 \%$ of an Australian horse population was reported lame by their owners (Lesimple et al., 2012). In the study of Murray et al. (2010), it appeared that older, as well as taller, horses were more likely to have had an episode of lameness in the last 2 yr. In the present study, neither taller horses nor older ones were more at risk for lameness compared to their smaller or younger counterparts. However, it was found that competition horses were less often lame compared to horses used for other purposes, for instance, recreation and instruction. In the study of Cooper and Albentosa (2005) and in the study of Murray et al. (2010) back problems were strongly associated with lameness. One major factor contributing to back pain is thought to be bad saddle fitting and weight distribution of the rider (De Cocq et al., 2004, 2009). It was remarkable to find in our data set that regular visits from a saddle maker was associated with a higher risk for horses being lame. Since the results of epidemiological research do not necessarily point to causal relationships between clinical problems and potential risk factors, there might be factors other than those that were recorded in our study underlying the association between visits of the saddle maker and horses being lame. It might be, for example, that one reason for a farm to be frequently visited by the saddle maker is the presence of horses with lameness problems.

Unfortunately, because discomfort or pain in horses is frequently not recognized by the owner, horses experiencing pain often continue to be ridden. The main complaints by riders and trainers are sudden exercise intolerance and poor performance, but also a sudden and progressive change of temperament can occur (i.e., animals become aggressive [Cauvin, 1997]). Recently, several studies have investigated the seriousness of back pain problems in horses. Prevalences range from $4-22 \%$, according to questionnaires, to $26-85 \%$ when examined with specialized equipment (sEMG measurements) and manual palpation by an experienced chiropractor (Landman et al., 2004; Lesimple et al., 2012). In the present study, the prevalence of back problems 
was $31 \%$; this is within the wide range of prevalences found with clinical examination.

Risk factors reported as being associated with back pain problems are riding techniques, saddle fitting, and weight distribution (De Cocq et al., 2004; Lesimple et al., 2012; Greve and Dyson, 2013). In the present study, several other factors appeared to form a risk factor for back problems. Horses with many different riders (e.g., horses in riding schools), and hence possibly different riding skills, riders' attributes, and riding level, had a higher risk to develop back pain problems.

As compared to other species assessed with a Welfare Quality protocol, horses are not only housed, fed, and transported, but are also used by humans in many different ways. With over 460,000 people in the Netherlands being active in horseback riding, it has become the third largest sport in the country (KNHS, 2012). For riding and driving, horses are haltered and most of the time also wear bits. Obviously, poorly fitted halters, poorly fitted bits, strong hands on the reins, and incompetent riding skills can cause discomfort in the horse's mouth, possibly resulting in irregularities in mouth corners and at the bars (Tell et al., 2008; Quick and Warren-Smith, 2009; Cook, 2011). Although these problems are very well recognized in the industry, the seriousness, in terms of prevalence, has not been documented very well. In this study, $18.5 \%$ of the horses were assessed with irregularities in the mouth corners. These included old and fresh wounds. A much smaller percentage of the horses $(3.4 \%)$ showed irregularities at the bars. Not surprisingly, the risk factor analyses revealed that there is a significant relation between irregularities in the mouth corners and being worked.

The assessment of horse welfare warrants a multidisciplinary approach in which risk factors for welfare problems can be clearly identified to improve the welfare standard and advise horse owners to adjust practices and management accordingly. Within this study we did not ask or investigate possible management or intervention measures that horse owners or caretakers have taken to increase the possible welfare deterioration because of clinical conditions. In the present large-scale study, welfare problems, under domestic horse husbandry conditions in the Netherlands, have been assessed as objectively as possible, with a minimum collection of data via questionnaires. The prevalences of health problems and the identified risk factors are valid for the horses in the present study in which farms voluntarily participated. Furthermore, the results may provide the basis for horse welfare and health programs on farm and horse industry levels. Moreover, due to the large scale of the study, the horse owners who participated in the study have gained a greater awareness on horse welfare aspects.

\section{LITERATURE CITED}

Becvarova, I., and R. S. Pleasant. 2012. Managing obesity in pasturebased horses. Compend. Contin. Educ. Vet. 34:E1-E4.

Bland, I., and J. Hill. 2011. Tackling dog obesity by tackling owner attitudes. CAB Rev. Perspect. Agric. Vet. Sci. Nutr. Nat. Resour. 6:1-7.

Blokhuis, H. J., R. B. Jones, R. Geers, M. Miele, and I. Veissier. 2003. Measuring and monitoring animal welfare: Transparency in the food product quality chain. Anim. Welfare 12:445-455.

Breslow, N. E., and D. G. Clayton. 1993. Approximate inference in generalized linear mixed model. J. Am. Stat. Assoc. 88:9-25.

Burn, C. C., T. L. Dennison, and H. R. Whay. 2010. Relationships between behaviour and health in working horses, donkeys, and mules in developing countries. Appl. Anim. Behav. Sci. 126:109-118.

Burn, C. C., J. C. Pritchard, and H. R. Whay. 2009. Observer reliability for working equine welfare assessment: Problems with high prevalences of certain results. Anim. Welfare 18:177-187.

Carroll, C. L., and P. J. Huntington. 1988. Body condition scoring and weight estimation of horses. Equine Vet. J. 20:41-45.

Carter, R. A., K. H. Treiber, R. J. Geor, L. Douglass, and P. A. Harris. 2009. Prediction of incipient pasture-associated laminitis from hyperinsulinaemia, hyperleptinaemia and generalised and localised obesity in a cohort of ponies. Equine Vet. J. 41:171-178.

Casey, R. A. 2002. Clinical problems associated with the intensive management of performance horses. In: N. Waren, editor, The Welfare of Horses. Kluwer Acad, Dordrecht, the Netherlands. p. 19-44.

Cauin, E. 1997. Assessment of back pain in horses. In Pract. 19:522-533.

Clarke, A. F. 1987. A review of environmental and host factors in relation to equine respiratory disease. Equine Vet. J. 19:435-441.

Cole, F. L., D. R. Hodgson, S. W. J. Reid, and D. J. Mellor. 2005. Owner-reported equine health disorders: Results of an Australiawide postal survey. Aust. Vet. J. 83:490-495.

Cook, W. R. 2011. Damage by the bit to the equine interdental space and second lower premolar. Equine Vet. Educ. 23:355-360.

Cooper, J. J., and M. J. Albentosa. 2005. Behavioural adaptation in the domestic horse: Potential role of apparently abnormal responses including stereotypic behaviour. Livest. Prod. Sci. 92:177-182.

Couëtil, L. L., A. M. Hoffman, J. Hodgson, V. Buechner-Maxwell, L. Viel, J. L. N. Wood, and J. P. Lavoie. 2007. Inflammatory airway disease of horses. J. Vet. Intern. Med. 21:356-361.

De Cocq, P., H. M. Clayton, K. Terada, M. Muller, and J. L. van Leeuwen. 2009. Usability of normal force distribution measurements to evaluate asymmetrical loading of the back of the horse and different rider positions on a standing horse. Vet. J. 181:266-273.

De Cocq, P., P. R. Van Weeren, and W. Back. 2004. Effects of girth, saddle and weight on movements of the horse. Equine Vet. J. 36:758-763.

Engel, B., and A. Keen. 1994. A simple approach for the analysis of generalised linear mixed models. Stat. Neerl. 48:1-22.

Frank, N. 2009. Equine Metabolic Syndrome. J. Equine Vet. Sci. 29:259-267.

Galantino-Homer, H. L., and J. B. Engiles. 2012. Insulin resistance and laminitis in broodmares. J. Equine Vet. Sci. 32:680-688.

Geor, R. J. 2008. Metabolic predisposition to laminitis in horses and ponies: Obesity, insulin resistance and metabolic syndromes. J. Equine Vet. Sci. 28:753-759.

German, A. 2010. Obesity in companion animals. In Pract. 32:42-50.

Goedhart, P. W. 2011. Procedure VSEARCH. In: P. W. Goedhart and J. T. N. M. Thissen, editors, Biometris GenStat Procedure Library Manual. 14th ed. Biometris Rep. 19.08.11.

Greve, L., and S. Dyson. 2013. The horse-saddle-rider interaction. Vet. J. 195:275-281. 
Halliwell, R. E. W., B. C. McGorum, P. Irving, and P. Dixon. 1993. Local and systemic antibody production in horses affected with chronic obstructive pulmonary disease. Vet. Immunol. Immunopathol. 38:201-215.

Hotchkiss, J. W., S. W. J. Reid, and R. M. Christley. 2007. A survey of horse owners in Great Britain regarding horses in their care. Part 1: Horse demographic characteristics and management. Equine Vet. J. 39:294-300.

Ireland, J. L., P. D. Clegg, C. M. McGowan, S. A. McKane, K. J. Chandler, and G. L. Pinchbeck. 2012. Comparison of owner-reported health problems with veterinary assessment of geriatric horses in the United Kingdom. Equine Vet. J. 44:94-100.

Johnson, P. J., C. E. Wiedmeyer, N. T. Messer, and V. K. Ganjam. 2009. Medical implications of obesity in horses-lessons for human obesity. J. Diabetes Sci. Technol. 3:163-174.

Kenward, M. G., and J. H. Roger. 1997. Small sample inference for fixed effects from restricted maximum likelihood. Biometrics 53:983-997.

KNHS. 2012. Paardenbezit onder paardensporters in Nederland fors toegenomen. www.knhs.nl/news.asp?id=24268.

Landman, M. A. A. M., J. A. de Blaauw, P. R. van Weeren, and L. J. Hofland. 2004. Field study of the prevalence of lameness in horses with back problems. Vet. Rec. 155:165-168.

Lesimple, C., C. Fureix, E. De Margerie, E. Seneque, H. Menguy, and M. Hausberger. 2012. Towards a postural indicator of back pain in horses (Equus caballus). PLoS One 7:E44604.

Lesimple, C., C. Fureix, H. Menguy, and M. Hausberger. 2010. Human direct actions may alter animal welfare, a study on horses (Equus callabus). PLoS One 5:E10257.

McGowan, T. W., G. Pinchbeck, C. J. C. Phillips, N. Perkins, D. R. Hodgson, and C. M. McGowan. 2010. A survey of aged horses in Queensland, Australia. Part 1: Management and preventive health care. Aust. Vet. J. 88:420-427.
Murray, R. C., J. M. Walters, H. Snart, S. J. Dyson, and T. D. H. Parkin. 2010. Identification of risk factors for lameness in dressage horses. Vet. J. 184:27-36.

NEWC. 2009. Equine Industry Welfare Quidelines Compendium for Horses, Ponies and Donkeys. 3rd ed. Natl. Equine Welfare Counc., Warwickshire, UK.

Pritchard, J. C., A. C. Lindberg, D. C. J. Main, and H. R. Whay. 2005. Assessment of the welfare of working horses, mules and donkeys, using health and behaviour parameters. Prev. Vet. Med. 69:265-283.

Quick, J. S., and A. K. Warren-Smith. 2009. Preliminary investigations of horses' (Equus caballus) responses to different bridles during foundation training. J. Vet. Behav. Clin. Appl. Res. 4:169-176.

Robinson, N. E., W. Karmaus, S. J. Holcombe, E. A. Carr, and F. J. Derksen. 2006. Airway inflammation in michigan pleasure horses: Prevalence and risk factors. Equine Vet. J. 38:293-299.

Searle, S. R., G. Casella, and C. E. McCulloch. 1992. Variance Components. John Wiley and Sons, New York.

Tell, A., A. Egenvall, T. Lundström, and O. Wattle. 2008. The prevalence of oral ulceration in Swedish horses when ridden with bit and bridle and when unridden. Vet. J. 178:405-410.

Thatcher, C. D., R. S. Pleasant, R. J. Geor, and F. Elvinger. 2012. Prevalence of overconditioning in mature horses in Southwest Virginia during the summer. J. Vet. Intern. Med. 26:1413-1418.

VSNInternational. 2012. GenStat for Windows. 15th ed. VSN Int., Hemel Hempstead, UK.

Wheeler, R. G., R. M. Christley, and C. M. McGowan. 2002. Prevalence of owner-reported respiratory disease in Pony Club horses. Vet. Rec. 150:79-81.

Wyse, C. A., K. A. McNie, V. J. Tannahil, J. K. Murray, and S. Love. 2008. Prevalence of obesity in riding horses in Scotland. Vet. Rec. 162:590-591. 